\title{
A pilot case-control study on the alveolar bone density measurement in risk assessment for bisphosphonate-related osteonecrosis of the jaw
}

\author{
Y. Takaishi • T. Ikeo • M. Nakajima • T. Miki • T. Fujita
}

Received: 25 February 2009 / Accepted: 6 July 2009 / Published online: 25 August 2009

(C) The Author(s) 2009. This article is published with open access at Springerlink.com

\begin{abstract}
Summary Alveolar bone mineral density (BMD) measured by radiography standardized by aluminum step wedge pasted on the film and digitized by a computer system was significantly higher around osteonecrosis lesions than in control cases in a pilot case-control study. High alveolar bone density appears useful as a local risk factor for bisphosphonate-related osteonecrosis of the jaw (BRONJ). Introduction In an attempt to find a reliable test method predicting the occurrence of BRONJ in addition to various risk factors suggested, an increase of alveolar bone density near the necrotic lesions was found by computerized radiogrammetry using dental films pasted with an aluminum step wedge (Bone Right $\AA$, Dentalgraphic.Com Company, Himeji) in six cases of BRONJ.
\end{abstract}

\author{
Y. Takaishi $(\bowtie)$ \\ Takaishi Dental Clinic, \\ 3-32, Hojo-guchi, \\ Himeji 670-0935, Japan \\ e-mail: takaishi@dentalgraphic.com \\ Y. Takaishi $\cdot$ T. Ikeo \\ Department of Biochemistry, Osaka Dental University, \\ Osaka, Japan \\ M. Nakajima \\ Department of Oral and Maxillofacial Surgery, \\ Osaka Dental University, \\ Osaka, Japan \\ T. Miki \\ Department of Geriatric Medicine, Osaka City University, \\ Osaka, Japan \\ T. Fujita \\ Katsuragi Hospital, \\ Osaka, Japan
}

Methods The bone mineral density surrounding the osteonecrosis lesions showed distinctly higher density in BRONJ cases compared with age-matched controls. In one subject on bisphosphonate treatment in whom two extractions were simultaneously carried out, BRONJ occurred only at the location with extremely high alveolar bone density, but not at the other site with normal density.

Conclusion This method may be useful in detecting a rise of alveolar BMD frequently occurring near the necrotic lesion in subjects with impending risk for BRONJ.

Keywords Alveolar bone density (al-BMD) . Bisphosphonate - Dental extraction · Dental X-ray film . Jaw bone osteonecrosis

\section{Introduction}

As an ominous complication of the most effective and popular treatments of osteoporosis, bone metastasis, and bone tumors, bisphosphonate-related osteonecrosis of the jaw (BRONJ) emerged with the first report of 36 cases by Marx in 2003 [1]. BRONJ is typically manifested by spontaneous exposure of the jaw bone with pain and swelling. The delay in the healing of the alveolar bone after dental extraction or other surgical procedure along with gingival swelling and pus discharge characterizes its course. The American Association of Oral and Maxillofacial Surgeons and the American Society for Bone and Mineral Research defined BRONJ with three characteristics: (1) use of bisphosphonate at present or in the past, (2) exposure of the necrotic jaw bone for 8 weeks or longer, and (3) absence of history of radiation therapy on the jaw area $[2,3]$. 
Epidemiological and clinical risk factors such as intravenous injection of a large dose of bisphosphonate, use of potent nitrogen-containing bisphosphonate at higher doses and over longer period, presence of cancer, diabetes mellitus, and other debilitating conditions, and treatment with irradiation or corticosteroid were also pointed out [4-6]. Surgical intervention including dental extraction appears to represent an imminent, almost prerequisite risk [7]. No effective tests predicting the occurrence of BRONJ are yet available. Pronounced fall of CTX, a marker of bone resorption, evidently a bisphosphonate effect, was also reported to occur in some patients with BRONJ [8].

In the process of searching for a readily available screening method for the occurrence of BRONJ, a new radiogrammetric method on the alveolar bone mineral density was developed using aluminum step wedge, pasted on dental film, to characterize alveolar bone under imminent danger for BRONJ $[9,10]$.

\section{Materials and methods}

\section{Selection of the test subjects}

Subjects with pathologically established cases of BRONJ after dental extraction were selected for alveolar bone density measurement. All of them had been treated with bisphosphonates and exposed to systemic risk factors for BRONJ such as glucocorticoid treatment or infection. Controls represented all subjects with age difference less than \pm 2 years from among subjects under care by the authors without history of bisphosphonate treatment, irradiation, or dental surgical treatment, also subjected to alveolar BMD (al-BMD) measurement by Bone Right radiogrammetry.
Measurement of alveolar bone density

Dental X-ray films were taken and alveolar bone density at the root of the first mandibular premolar measured, as described elsewhere [9], using an originally designed image editing software (No. PCT/jp2004/010815). A line was drawn at the apex of the root, parallel to the boundary of the cement-enamel junction. Another line was drawn halfway between the cement-enamel junction and the apex of the root. Lines were then drawn perpendicular to those lines at the mesial and distal spaces of the first premolar. The X-ray film density in the area of the resulting rectangles was measured by first dividing the area into pixels with sides $1 / 1,524 \mathrm{~cm}$ in length. The brightness in each pixel was then compared with a scale consisting of 256 steps of brightness (Fig. 1).

In order to align and standardize the brightness and contrast among the X-ray pictures for comparison of the results of measurement among X-ray pictures taken on different occasions, an X-ray picture taken for a normal, healthy person (i.e., a 23-year-old woman having $100 \%$ bone mineral density in the example being described) was used as a reference. A histogram hist $[x]$ of a color bar on the reference picture was normalized according to Eq. 1. Then, the normalized histogram hist $[x]$ is substituted in Eqs. 2 and 3 to thereby calculate the brightness mean value, mean, and the standard deviation, SD, which are referred to as the reference mean value, RefMean, and the reference deviation, RefSD, respectively.

Similarly, for each of the pictures to be corrected, the histogram hist $[x]$ of its color bar is normalized and the brightness mean value and the SD for that picture calculated. Mean, the mean value of the brightness thus calculated, and SD, standard deviation, RefMean, the reference mean value, and RefSD, the reference deviation, are substituted in Eq. 4 to correct the respective pictures
Fig. 1 Geometry of alveolar bone measurement. a Aluminum step wedge for calibration. b Calibration of density between standard aluminum wedge and maximum/minimum density. c Defining the area of interest for the alveolar bone density

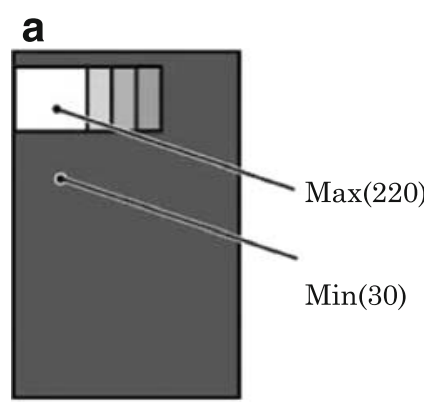

b
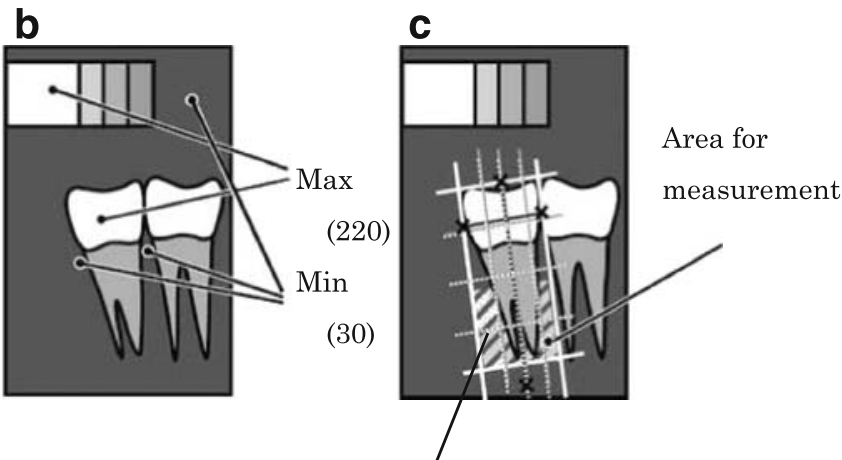

The area is divided into microsquares. 
Table 1 Comparison of al-BMD between cases of BRONJ and age-matched controls (seven cases each)

\begin{tabular}{|c|c|c|c|c|c|c|}
\hline Case no. & Age & Al-BMD & Control no. & Age & Al-BMD & Difference (Student's $t$ test) \\
\hline \multirow[t]{8}{*}{ Case 1} & \multirow[t]{8}{*}{75} & 184.1 & Control 1-1 & 70 & 73.0 & $* *$ \\
\hline & & 175.6 & $1-2$ & 75 & 57.6 & $t=6.73$ \\
\hline & & 184.5 & $1-3$ & 75 & 23.0 & $p=0.0001$ \\
\hline & & \multirow[t]{5}{*}{ (Mean 181.3) } & $1-4$ & 75 & 108.5 & \\
\hline & & & $1-5$ & 75 & 85.0 & \\
\hline & & & $1-6$ & 76 & 53.0 & \\
\hline & & & $1-7$ & 76 & 33.8 & \\
\hline & & & Mean \pm SD & $74.6 \pm 1.9$ & $61.9 \pm 29.5$ & \\
\hline \multirow[t]{8}{*}{ Case 2} & \multirow[t]{8}{*}{75} & 132.1 & Control 2-1 & 70 & 73.0 & $* *$ \\
\hline & & 123.6 & $2-2$ & 75 & 57.6 & $t=5.42$ \\
\hline & & 120.4 & $2-3$ & 75 & 23.0 & $p=0.0073$ \\
\hline & & (Mean 125.3) & $2-4$ & 75 & 108.5 & \\
\hline & & & $2-5$ & 75 & 85.0 & \\
\hline & & & $2-6$ & 76 & 53.0 & \\
\hline & & & $2-7$ & 76 & 33.8 & \\
\hline & & & Mean \pm SD & $74.6 \pm 1.9$ & $61.9 \pm 29.5$ & \\
\hline \multirow[t]{8}{*}{ Case 3} & \multirow[t]{8}{*}{61} & 150.2 & Control 3-1 & 60 & 78.8 & $\mathrm{~ns}$ \\
\hline & & 98.5 & $3-2$ & 60 & 81.2 & $t=1.86$ \\
\hline & & 84.7 & $3-3$ & 60 & 95.1 & $p=0.0991$ \\
\hline & & (Mean 111.1) & $3-4$ & 60 & 96.0 & \\
\hline & & & $3-5$ & 60 & 83.6 & \\
\hline & & & $3-6$ & 61 & 81.6 & \\
\hline & & & $3-7$ & 61 & 95.0 & \\
\hline & & & Mean \pm SD & $60 \pm 0$ & $87.3 \pm 17.2$ & \\
\hline \multirow[t]{8}{*}{ Case 4} & \multirow[t]{8}{*}{49} & 190.0 & Control 4-1 & 50 & 80.8 & $* *$ \\
\hline & & 189.1 & $4-2$ & 50 & 99.9 & $t=6.09$ \\
\hline & & 157.6 & $4-3$ & 51 & 58.4 & $p=0.003$ \\
\hline & & (Mean 178.9) & $4-4$ & 51 & 108.8 & \\
\hline & & & $4-5$ & 51 & 63.0 & \\
\hline & & & $4-6$ & 51 & 96.1 & \\
\hline & & & $4-7$ & 51 & 116.0 & \\
\hline & & & Mean \pm SD & $50.6 \pm 0.4$ & $89.0 \pm 22.2$ & \\
\hline \multirow[t]{7}{*}{ Case 5} & \multirow[t]{7}{*}{47} & 138.6 & Control 5-1 & 49 & 64.5 & $* *$ \\
\hline & & 152.5 & $5-2$ & 49 & 83.9 & $t=7.57$ \\
\hline & & 168.4 & $5-3$ & 48 & 66.1 & $p=0.0001$ \\
\hline & & (Mean 153.2) & $5-4$ & 48 & 91.2 & \\
\hline & & & $5-5$ & 46 & 63.5 & \\
\hline & & & $5-6$ & 45 & 93.2 & \\
\hline & & & Mean \pm SD & $47.1 \pm 1.8$ & $77.0 \pm 13.9$ & \\
\hline \multirow[t]{8}{*}{ Case 6} & \multirow[t]{8}{*}{60} & 214.1 & Control 6-1 & 60 & 78.8 & $* *$ \\
\hline & & 229.4 & $6-2$ & 60 & 81.2 & $t=18.81$ \\
\hline & & 200.5 & $6-3$ & 60 & 95.1 & $p<0.0001$ \\
\hline & & (Mean 214.7) & $6-4$ & 60 & 96.0 & \\
\hline & & & $6-5$ & 60 & 83.6 & \\
\hline & & & $6-6$ & 61 & 81.6 & \\
\hline & & & $6-7$ & 61 & 95.0 & \\
\hline & & & Mean \pm SD & $60.3 \pm 0.5$ & $87.3 \pm 7.7$ & \\
\hline \multirow[t]{3}{*}{ Total } & \multirow[t]{3}{*}{$61.2 \pm 12.1$} & \multirow[t]{3}{*}{$160.8 \pm 39.4$} & & $61.3 \pm 10.8$ & $77.7 \pm 22.6$ & $* *$ \\
\hline & & & & & & $t=7.10$ \\
\hline & & & & & & $p<0.0001$ \\
\hline
\end{tabular}


Table 1 (continued)

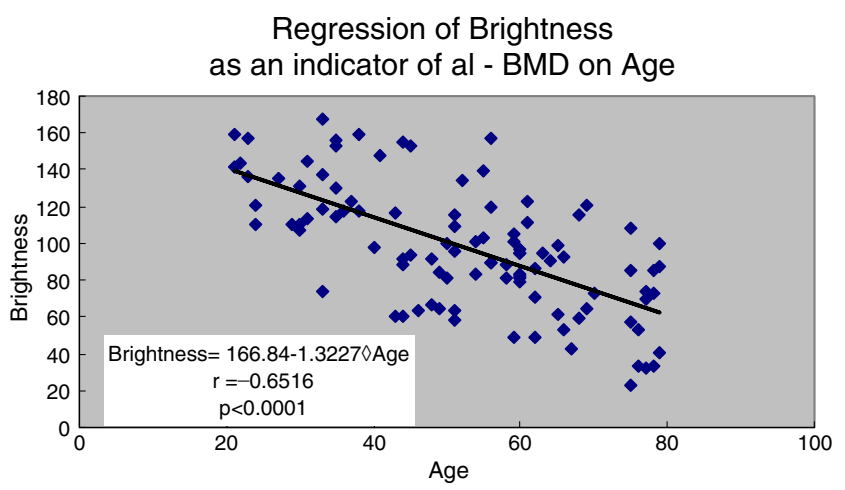

Student's $t$ test revealed significant difference between each pair of cases 1,2, 4, 5, and 6 and controls, but not between case 3 and controls. Overall statistical analysis showed a highly significant difference at $p=0.0001$

$(* * \mathrm{p}<0.01)$

with respect to their brightness and contrast and to obtain corrected brightness value $Y^{\prime}(i, j)$ for each picture.

$\operatorname{hist}[x]=\frac{\operatorname{Num}[x]}{\text { TotalNum }}$

where $x(0 \leq x \leq 255)$ is gradation, $\operatorname{Num}[x]$ is the number of pixels for the gradation $x$ in the color bar, and TotalNum is the total number of pixels of the color bar.

Mean $=\sum_{x=0}^{255}\{x \times \operatorname{hist}[x]\}$

$\mathrm{SD}=\sqrt{\sum_{x=0}^{255}\left\{(x-\text { Mean })^{2} \times \operatorname{hist}[x]\right\}}$

$Y^{\prime}(i, j)=\frac{\operatorname{RefSD}}{S D} \times\{Y(i, j)-$ Mean $\}+$ RefMean

where $Y(i, j)$ is the brightness value of a picture before correction is provided and $(i, j)$ is a coordinate of a given pixel.

Using a thin adhesive aluminum step wedge pasted on the X-ray film, pictures of regions around the first right mandibular premolar tooth were taken, with a special caution to place the X-ray tube vertical to the film. The dental X-ray film after exposure is then taken into a laptop computer using a scanner. Data and histogram of the al-BMD were recorded on the screen in a few minutes using a software (Bone Right $\AA$, Dentalgraphic.Com Company) $[9,10]$. This technique may also be applied similarly to any tooth in a panorama film covering the whole series of the teeth in an individual. As shown in Table 1, al-BMD showed a significantly negative coefficient regression on age.
In summary, this new method of standardization of the results of measurement of alveolar bone density made it possible to compare the brightness data accurately between films taken with time intervals. The use of aluminum step wedge is not for direct comparison of brightness between films but for normalization and standardization of the data by computation; as the result, $\mathrm{cv}$ of $1.94 \%$ was achieved on measurement of al-BMD in 20 subjects at 2-week intervals.

\section{Case report and results of measurement}

Case 1: BRONJ occurrence adjacent to high al-BMD region but not adjacent to normal density on double extraction

The first case is a 75-year-old woman with multiple myeloma treated with $10 \mathrm{mg}$ monthly intravenous incadronate for 5 years along with dexamethasone, ranimustine, vincristine, and interferon. In June 2006, right maxillary canine, right maxillary first premolar, and left mandibular first molar were extracted.

As shown in Fig. 2a, a dental X-ray film view revealed disappearance of the trabecular structure of the mandible. Pathological findings were characteristic of BRONJ with scarcely any osteocytes visible in the area involved; a radioopaque area surrounded by relatively radiolucent area interspersed with bacterial flora and inflammatory granulation tissue, indicating chronic suppurative osteomyelitis. The bone mineral density was extremely high around the BRONJ lesion, $181.3 \pm 5.0(6,7,8$, means $\pm \mathrm{SD}, N=3)$, far exceeding the mean bone mineral density in healthy young subjects and significantly higher than the density around the non-necrotic areas, $146.4 \pm 19.1(1,2,3$, mean $\pm \mathrm{SD}, N=3)$ where no BRONJ occurred (Fig. 2a). Although right maxillary canine and first premolar as well as left mandibular first molar were also extracted along with the 
Fig. 2 a Case 1, 75-year-old female. Panorama X-ray film and results of al-BMD measurement. No osteonecrosis is noted around the first premolar of the right mandible [1-3], with high al-BMD values $130-167$. At sites 9 and 10 , on the contralateral side with extraction, no BRONJ occurred and al-BMD stayed as low as 30-42. At site 5 exhibiting chronic suppurative osteomyelitis alone, al-BMD stayed within normal range, 120. At sites 6, 7, and 8 around BRONJ which occurred after extraction, extremely high alBMD of 175-184 was noted. b Case2, 75-year-old female. Osteonecrosis is noted around the right mandibular molar and premolar regions 5,6 , and 8 around the site of extraction with higher al-BMD than regions 1, 2, 3, and 7 elsewhere. c Case 3, a 61-year-old female exhibited an extremely high alBMD of 150 after intravenous zoledronate at site 2 around the BRONJ lesion which followed an extraction, but normal density of 84-98 around the neighboring teeth

\section{a}

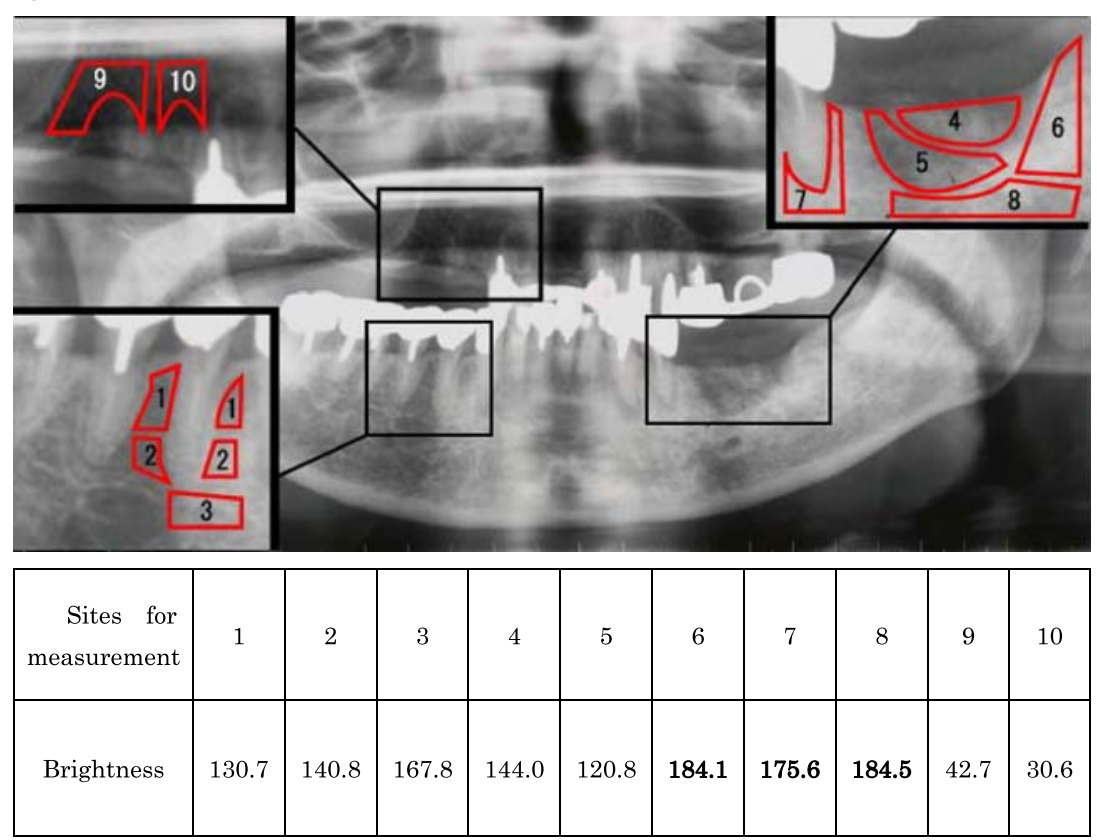

b
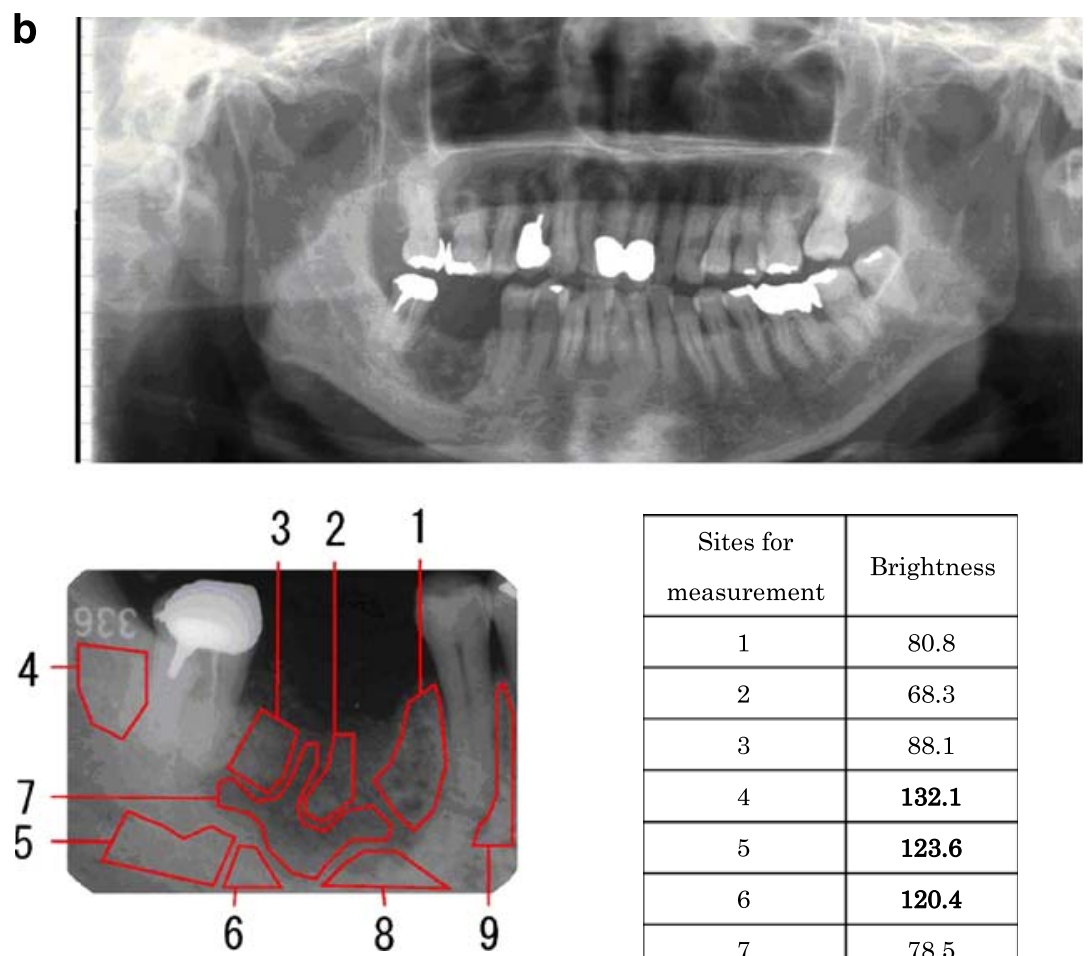

left mandibular molar, BRONJ developed only around the left first molar of the mandible. Since the al-BMD around the right canine and first premolar of the maxilla was low [30.6 and $42.7(9,10)]$, unusually high local al-BMD are apparently associated with BRONJ. Detection and evaluation of locally high BMD in the jaw bone apparently made an early detection of BRONJ possible. Apparently, dental extraction and accompanying tissue damage, infection, hemorrhage, etc. accelerate or provoke infectious or necrotic process in the development of BRONJ. Seven age-matched control cases showed al-BMD of $61.9 \pm 29.5$, significantly lower than in this case $(p<0.0001)$ as shown in Table 1. 
Fig. 2 (continued)
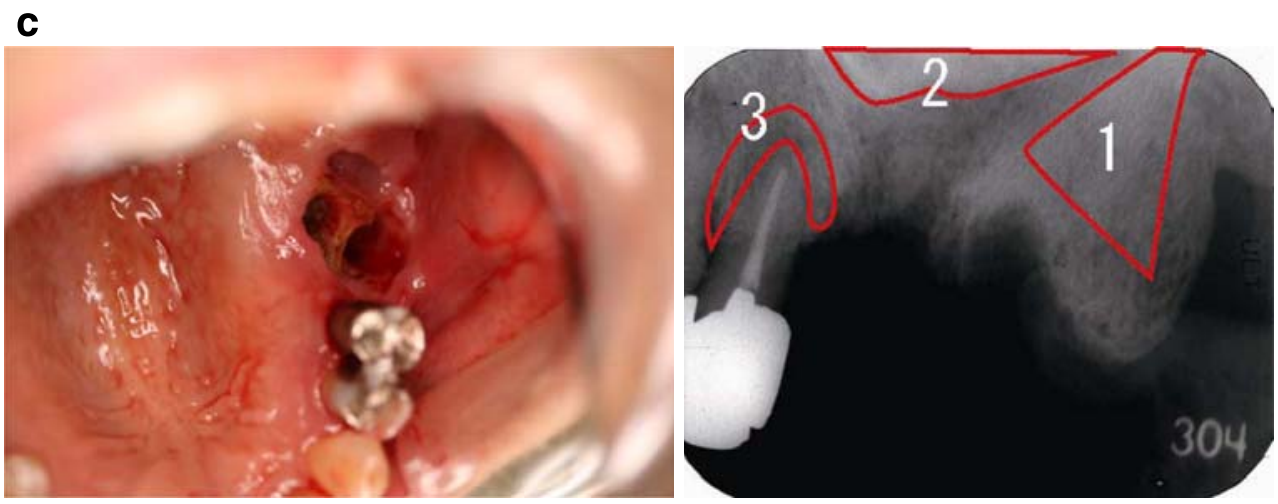

\begin{tabular}{|c|c|c|c|}
\hline $\begin{array}{c}\text { Sites for } \\
\text { measurement }\end{array}$ & 1 & 2 & 3 \\
\hline Brightness & $\mathbf{8 4 . 7}$ & $\mathbf{1 5 0 . 4}$ & $\mathbf{9 8 . 5}$ \\
\hline
\end{tabular}

Case 2: BRONJ following oral alendronate treatment, $5 \mathrm{mg}$ daily for 6 years, for osteoporosis after 1-year corticosteroid treatment for rheumatic polymyalgia in a 75-year-old female

On initial examination on January 11, 2008, compression of right mandibular molar region elicited tenderness and pus discharge.

Extraction in October was followed by poor recovery. In January 2008, sequestrum was removed and BRONJ noted on pathological examination. Significantly higher al-BMD was also noted around the BRONJ lesion (132.1, 123.6, 120.4) than other sites and in control cases (Table 1 and Fig. 2b).

Case 3: BRONJ following intravenous zoledronate treatment of metastasizing breast cancer

BRONJ appeared in a 61-year-old female carrying breast cancer with bone and liver metastases on dental extraction on May 29, 2007 after intravenous zoledronate (4 mg/ month) over a period of 1 year and 4 months. On initial examination on September 10, 2007, the site of extraction, left upper first molar, was surrounded by a region with a high bone density, 150.4 versus 84.7 , and 98.5 brightness in the corresponding part of the alveolar bone under the two neighboring teeth (Fig. 2c). Washing of the oral cavity is still continued at present. Higher al-BMD was noted than in other sites and control cases (Table 1).

Case 4: BRONJ and chronic suppurative periodontitis following intravenous pamidronate and zoledronate treatment for 17 months

A 49-year-old female with breast cancer with multiple bone metastases to the bone, treated with pamidronate from
March 2004 and 4 mg/month zoledronate from April 2006, was first seen on September 20, 2007. BRONJ appeared on August 8, 2007, manifested by spontaneous exposure of natural bone on the lingual side of the second molar of the left mandible. The bone density at the apical portion of the site of necrosis (190.0, 189.1, and 157.6) [1-3] was definitely higher than the corresponding site in adjacent tooth without necrosis (154.5 and 130.3) [5, 6] (Fig. 3a). These values were also significantly higher than these in seven age-matched controls (Table 1). In November 2007, recurrence of breast cancer and metastasis to the sternum was noted.

Case 5: BRONJ following intravenous zoledronate treatment of breast cancer

Case 5 is a 47-year-old female. Diagnosis of cancer of the right breast was made in November 2002 and bone metastases detected in April 2007. Zoledronate (4 mg/month) was given until March 2009. Wounds at bridge site noted in November 2008 over the first left mandibular molar tooth extracted at 20 years of age failed to respond to washing and local debridement. Osteomyelitis of the jaw related to bisphosphonate treatment was diagnosed. Significantly higher al-BMD (138.6, 152.5, and 168.4) was also noted around the BRONJ lesion than other sites and in control cases (Table 1 and Fig. 3b).

Case 6: BRONJ following intravenous zoledronate treatment of metastasizing breast cancer

A 60-year-old female with left breast cancer was found with multiple metastases to lymph nodes on February 6, 2008. Dexamethasone (ten times) and zoledronate (4 mg, 14 times) were given in February 2008 and March 2009. 
Fig. 3 a Case 4, a 49-year-old female manifested mainly by chronic suppurative periodontitis with BRONJ despite intravenous pamidronate and zoledronate and no tooth extraction. At the apical portion of the bone exposure site and neighboring legions, extremely high al-BMD of 157-190 was noted as shown. b Case 5, a 47-year-old female exhibited an extremely high al-BMD after intravenous zoledronate. At sites 3,6 , and 8 around BRONJ lesion, extremely high al-BMD of 168-138 was noted. c Case 6, a 60-year-old female exhibited an extremely high al-BMD after intravenous zoledronate. At sites 2, 3, and 4 around BRONJ lesion, extremely high al-BMD of 214-200 was noted
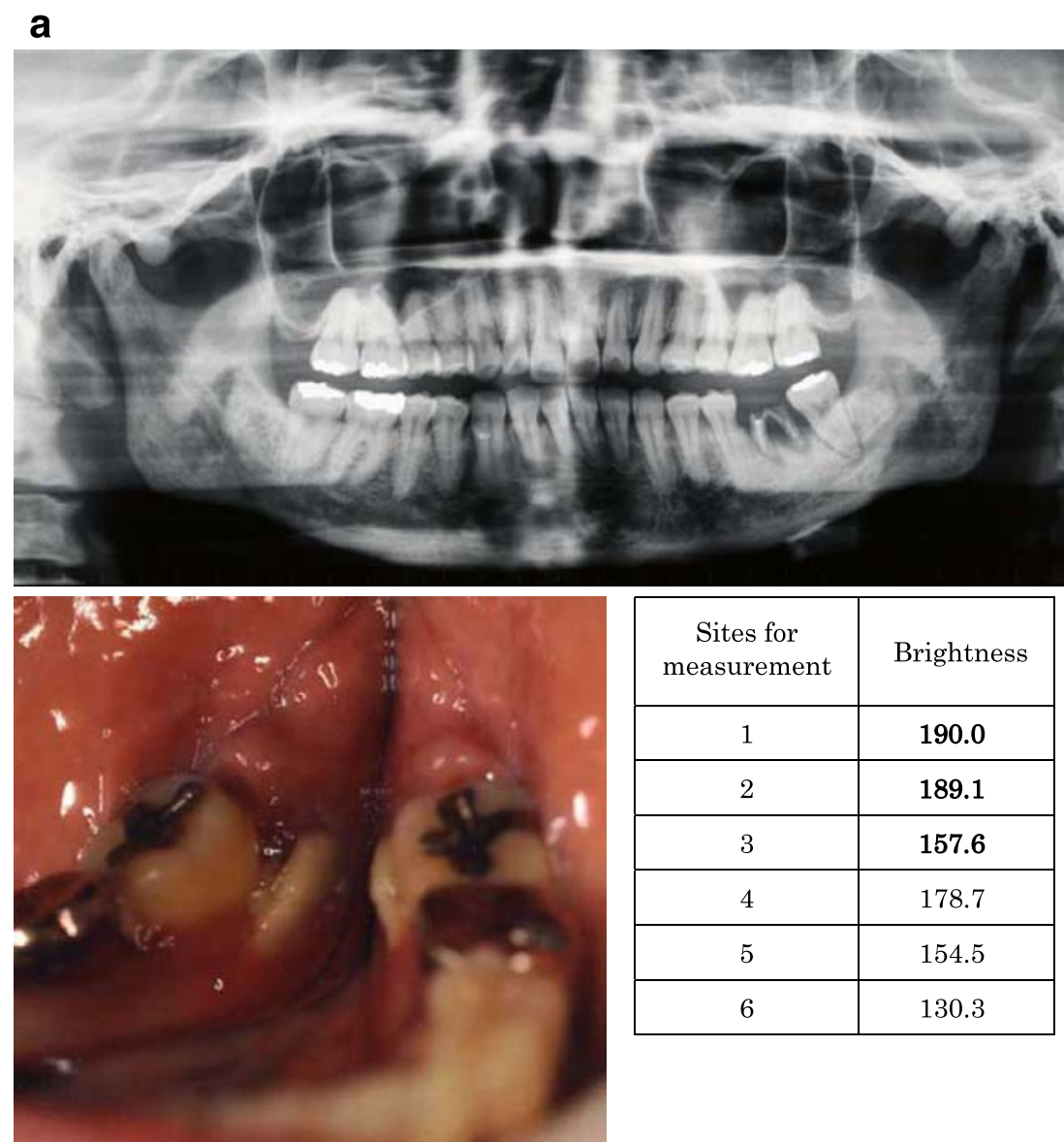

\begin{tabular}{|c|c|}
\hline $\begin{array}{c}\text { Sites for } \\
\text { measurement }\end{array}$ & Brightness \\
\hline 1 & $\mathbf{1 9 0 . 0}$ \\
\hline 2 & $\mathbf{1 8 9 . 1}$ \\
\hline 3 & $\mathbf{1 5 7 . 6}$ \\
\hline 4 & 178.7 \\
\hline 5 & 154.5 \\
\hline 6 & 130.3 \\
\hline
\end{tabular}

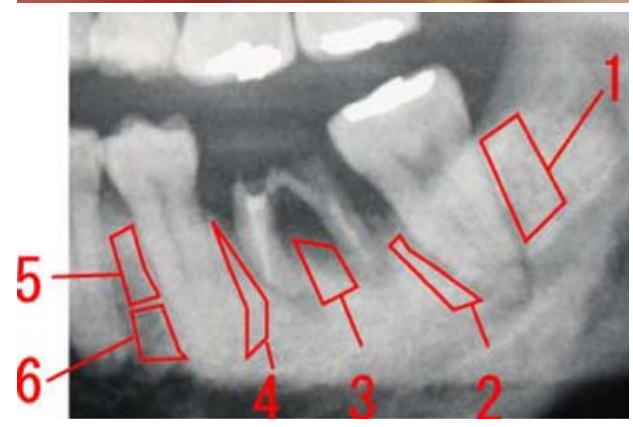

The second left mandibular molar tooth was extracted in April 2009. Delayed healing bone exposure and pus discharge led to diagnosis of BRONJ. Significantly higher al-BMD (214.1, 229.4, and 200.5) was also noted around the BRONJ lesion than other sites and in control cases (Table 1 and Fig. 3c).

\section{Reference case}

A case of radiation-associated necrosis of the jaw

Around the site of necrosis, 16,17 , and 18 as well as other sites, high al-BMD was found after radiation therapy; jaw bone necrosis occurred, though no bisphosphonate was given.

This study was approved by the Institutional Review Board of Japanese Association for the Promotion of State of Art in Medicine.

\section{Discussion}

Impressively high al-BMD around the BRONJ lesion is summarized in Table 1 . Highly statistically significant difference was found in individual cases as well as the whole series. It was especially noteworthy that BRONJ occurred only near the site with high al-BMD despite two similar dental extractions. 
Fig. 3 (continued)

b

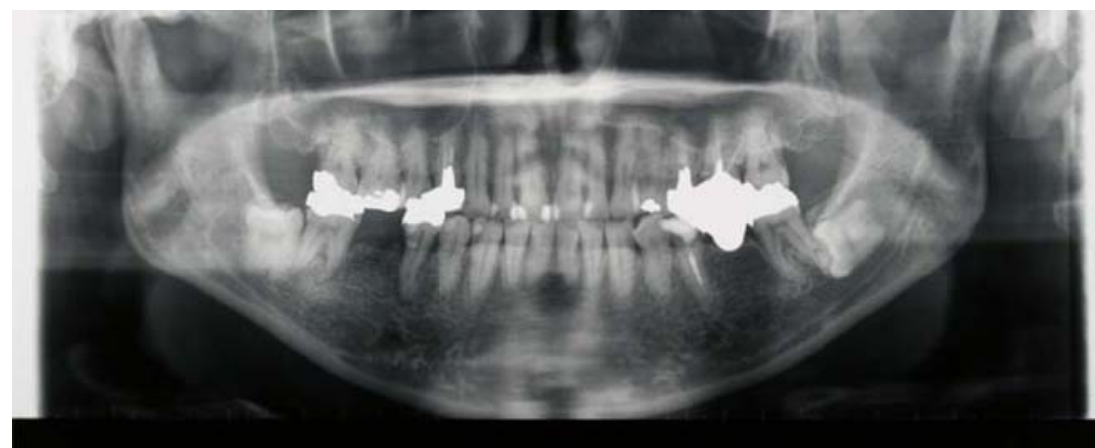

\begin{tabular}{|c|c|}
\hline $\begin{array}{c}\text { Sites for } \\
\text { measurement }\end{array}$ & Brightness \\
\hline 1 & 107.05 \\
\hline 2 & 138.1 \\
\hline 3 & $\mathbf{1 3 8 . 6}$ \\
\hline 4 & 59.0 \\
\hline 5 & 116.3 \\
\hline 6 & $\mathbf{1 5 2 . 5}$ \\
\hline 7 & 140.3 \\
\hline 8 & $\mathbf{1 6 8 . 4}$ \\
\hline 9 & 176.7 \\
\hline
\end{tabular}

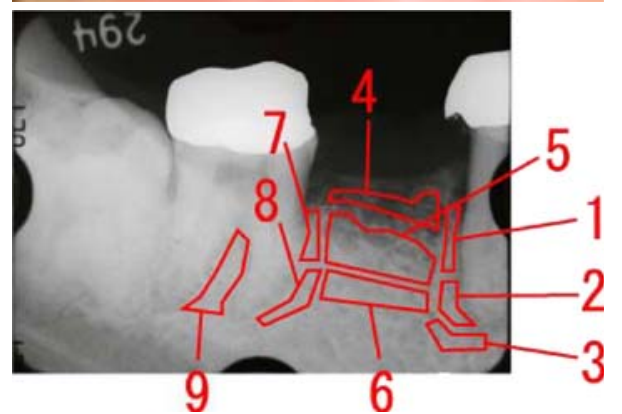

The computerized alveolar bone densitometry using dental X-ray film appears to be handy and useful to detect rises of local alveolar bone density with reference to the occurrence of BRONJ, as suggested by the six cases presented. In addition to the increase of jaw bone cortical thickness and suppression of bone turnover, local increases of alveolar bone density appears to contribute to BRONJ possibly through compromised circulation and physicochemical overload. Fall of the level of bone turnover may suppress defense reaction against external stimuli. Restricted angiogenesis may also occur along with osteosclerosis, leading to ischemia and poor nutritional supply interfering with wound healing process. On the other hand, the increase of density may suggest a response to nearby necrotic process already started to be aggravated, completing the necrosis in the response to the invasive procedure.
Radiation therapy also increases al-BMD. A 54-year-old male (reference case) underwent radiation therapy for cancer of the tongue on March 2, 2004 and given 20 courses of irradiation over a period of 2 months. Surgery for the cancer was performed in May 2005. Osteonecrosis of the jaw appeared on extraction of first molar of the right mandible on February 2007 at another dental clinic, with persistent bone exposure. On May 18, 2007, alveolar bone density was measured on dental and panorama X-ray film. High bone density of 171 to 191 brightness was noted throughout this period. Al-BMD at corresponding site in this case was as high as in case 1 , probably indicating a local risk for osteonecrosis of the jaw regardless of the cause (Fig. 4).

BRONJ is apparently a multi-factorial disease caused by systemic and local factors. As is evident from the discussion above, the present method using dental X-ray 
Fig. 3 (continued)

C
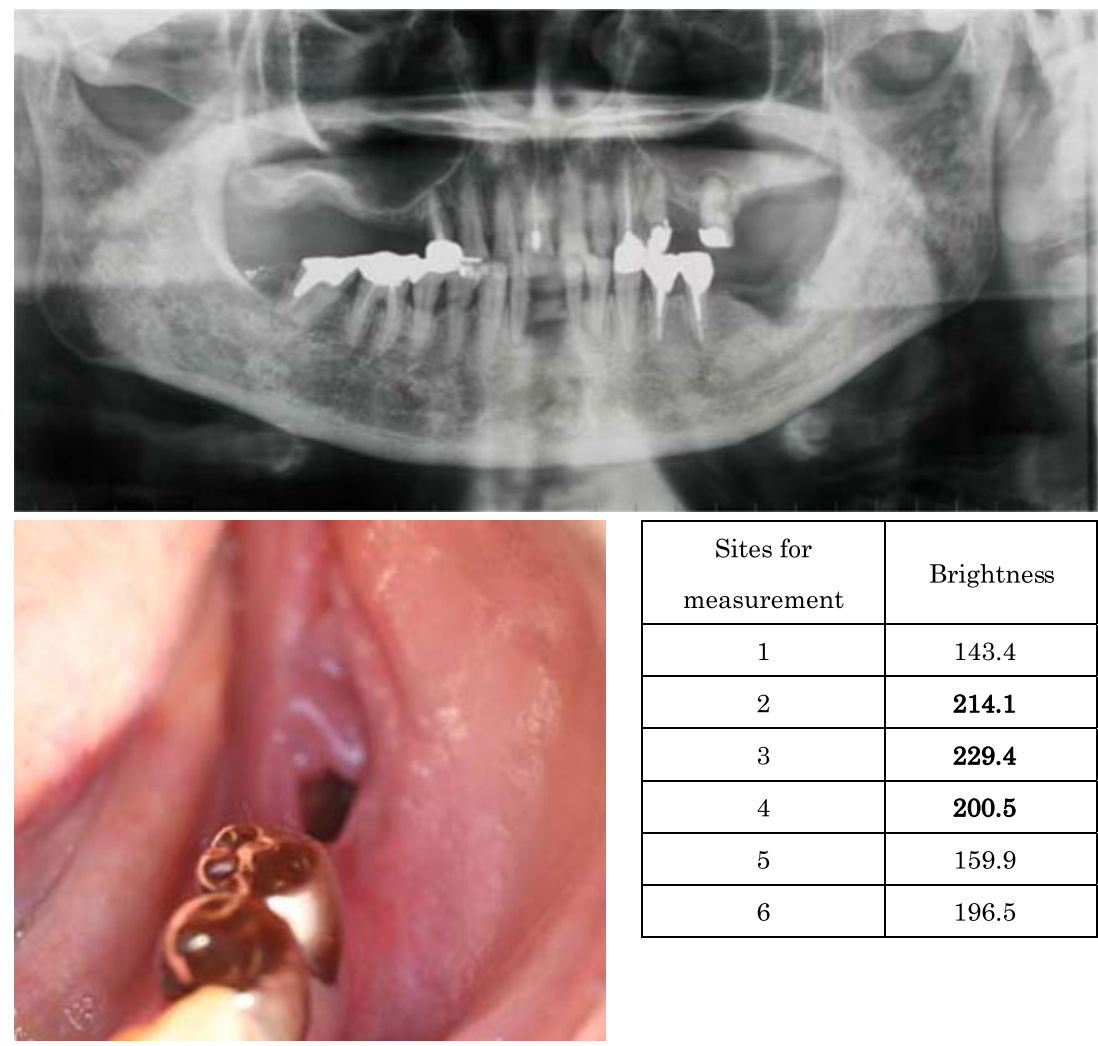

\begin{tabular}{|c|c|}
\hline $\begin{array}{c}\text { Sites for } \\
\text { measurement }\end{array}$ & Brightness \\
\hline 1 & 143.4 \\
\hline 2 & $\mathbf{2 1 4 . 1}$ \\
\hline 3 & $\mathbf{2 2 9 . 4}$ \\
\hline 4 & $\mathbf{2 0 0 . 5}$ \\
\hline 5 & 159.9 \\
\hline 6 & 196.5 \\
\hline
\end{tabular}

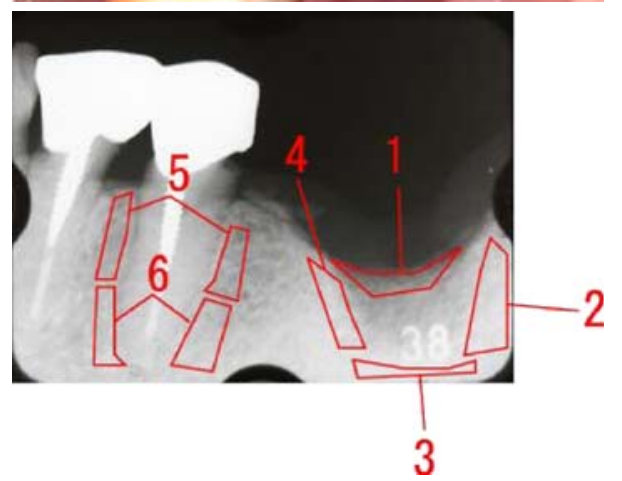

film with aluminum step wedge pasted makes it possible to measure alveolar bone mineral density at selected sites of the alveolar bone quantitatively with a higher sensitivity and reproducibility, unlike observation of panoramic X-ray film of the whole series of teeth only providing an overview or general impression. Since jaw bone has a high turnover, with a tendency of accumulation of microdamage and development of hard, brittle, and inactive bone in response to bisphosphonate therapy, detection of increased alveolar bone density may be especially useful to detect appearance of bone quality, which may be one of the factors causing BRONJ.

Pronounced fall of CTX, a bone resorption marker, to less than $100 \mathrm{pg} / \mathrm{ml}$ was pointed out by Marx et al. [8] as a systemic risk factor for BRONJ. Bisphosphonates increase
BMD through inhibition of osteoclastic bone resorption as indicated by a reduction of circulating bone resorption marker such as CTX. It is thus understandable that the larger the dose becomes, the more serum CTX falls. In view of the association of very low serum CTX with occurrence of BRONJ, excessive fall of serum CTX may serve as one of the risk factors for BRONJ. Such systemic marker of bisphosphonates, however, may not directly express local bone changes directly influencing the occurrence of BRONJ, taking into consideration factors such as bone quality and circulation in response to regional toxic effect of bisphosphonate. Measurement of the local al-BMD may therefore be more valuable as a predictor for BRONJ than systemic or circumstantial risk factors. 
The limitation of this case-control study consists in its pilot nature. The number of cases is also small. A prospective planned approach is desirable and a simple increase of the number of cases would not add to the reliability. This study, nevertheless, would suggest a usefulness of the new simple computerized alveolar bone density measurement using dental X-ray film. Attempts are also in progress to improve accuracy of the data by introducing thickness factor to simulate true three-dimensional density instead of the current two-dimensional projective density on the X-ray film. Prospective and systematic evaluation of al$\mathrm{BMD}$ with reference to the occurrence of BRONJ is in order to test the significance of high al-BMD as a local risk factor for BRONJ.
Fig. 4 Reference case, a 54year-old male with osteonecrosis of the jaw following radiation and tooth extraction exhibited high al-BMD values of 159-207 including the site around extraction and development of osteonecrosis of the jaw
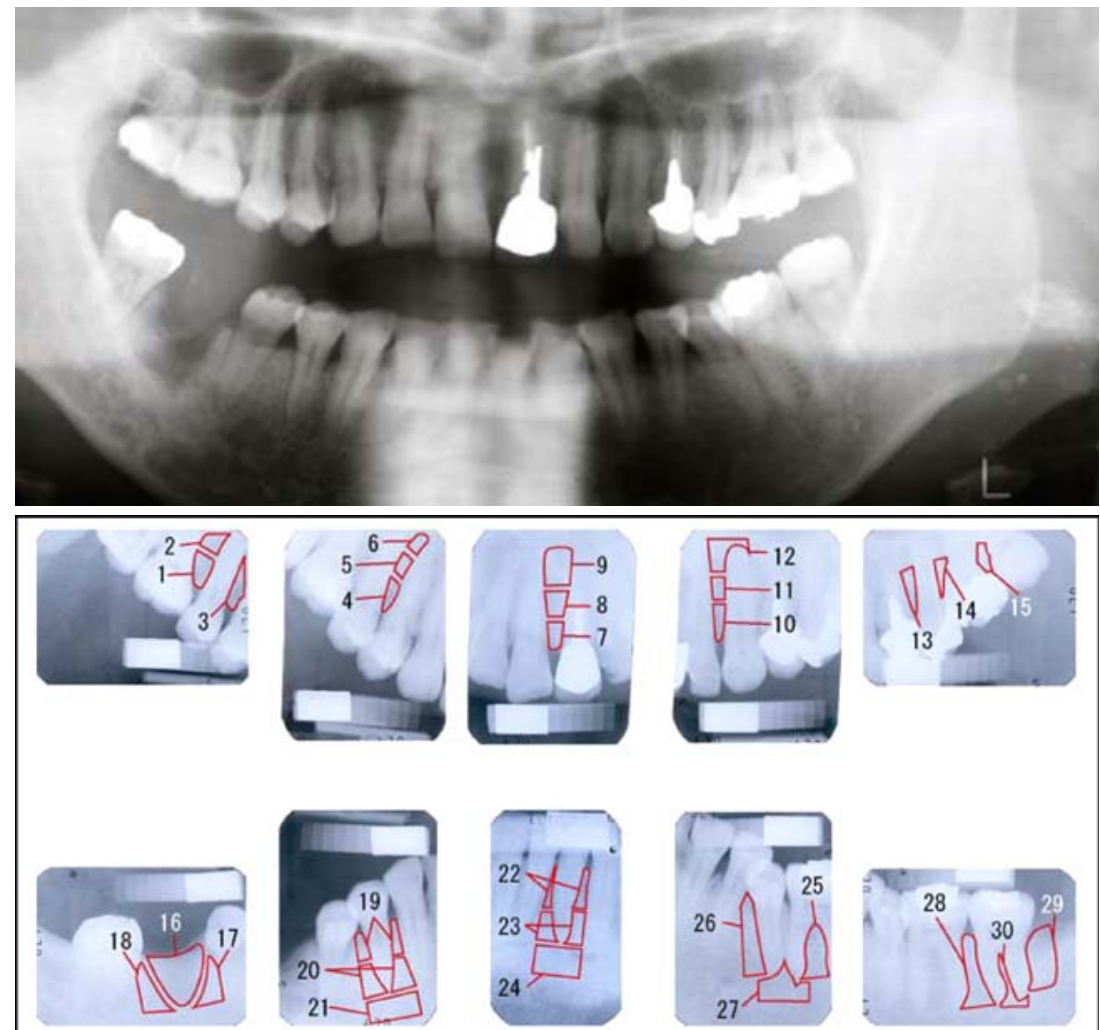

\begin{tabular}{|c|c|c|c|}
\hline $\begin{array}{c}\text { Sites for } \\
\text { measurement }\end{array}$ & $\begin{array}{c}\text { Brightness } \\
\text { Mean } \pm \text { SD }\end{array}$ & $\begin{array}{c}\text { Sites for } \\
\text { measurement }\end{array}$ & $\begin{array}{c}\text { Brightness } \\
\text { Mean } \pm \text { SD }\end{array}$ \\
\hline 1 & $175.1 \pm 6.3$ & 16 & $171.8 \pm 21.7$ \\
\hline 2 & $191.1 \pm 7.0$ & 17 & $191.0 \pm 5.8$ \\
\hline 3 & $165.7 \pm 11.9$ & 18 & $191.6 \pm 4.0$ \\
\hline 4 & $163.7 \pm 9.7$ & 19 & $185.4 \pm 14.6$ \\
\hline 5 & $189.9 \pm 6.2$ & 20 & $203.5 \pm 4.2$ \\
\hline 6 & $195.9 \pm 4.0$ & 21 & $197.1 \pm 3.9$ \\
\hline 7 & $181.4 \pm 7.7$ & 22 & $138.2 \pm 8.4$ \\
\hline 8 & $187.1 \pm 7.5$ & 23 & $160.6 \pm 10.0$ \\
\hline 9 & $191.5 \pm 4.4$ & 24 & $179.2 \pm 7.3$ \\
\hline 10 & $167.9 \pm 9.7$ & 25 & $174.4 \pm 7.1$ \\
\hline 11 & $186.1 \pm 6.1$ & 26 & $178.7 \pm 6.4$ \\
\hline 12 & $215.0 \pm 3.5$ & 27 & $164.1 \pm 4.4$ \\
\hline 13 & $188.1 \pm 9.0$ & 28 & $188.8 \pm 4.2$ \\
\hline 14 & $200.1 \pm 3.7$ & 29 & $189.1 \pm 6.8$ \\
\hline 15 & $203.6 \pm 3.2$ & 30 & $190.5 \pm 4.0$ \\
\hline
\end{tabular}


Open Access This article is distributed under the terms of the Creative Commons Attribution Noncommercial License which permits any noncommercial use, distribution, and reproduction in any medium, provided the original author(s) and source are credited.

\section{Conflicts of interest None.}

\section{References}

1. Marx RE (2003) Pamidronate (Aredia) and zoledronate (Zometa) induced avascular necrosis of the jaw: a growing epidemic. J Oral Maxillofac Surg 61:1115-1117

2. Advisory Task Force on Bisphosphonate-Related Osteonecrosis of the Jaws (2007) Position paper of the American Association of Oral and Maxillofacial Surgeons on bisphosphonate-related osteonecrosis of the jaws. J Oral Maxillofac Surg 65:369-376

3. Editorial (2007) Bisphosphonate-associated osteonecrosis of the jaw: report of a task force of the American Society for Bone and Mineral Research. J Bone Miner Res 22:1479-1490
4. Dello Russo NM, Jeffcoat MK, Marx RE, Fugazzotto P (2007) Osteoporosis in the jaw of patients who are using oral bisphosphonates to treat osteoporosis. Int J Oral Maxillofac Implants 22:146-153

5. Bamias A, Kastritis E, Bamias C (2006) Osteonecrosis of the jaw in cancer after treatment with bisphosphonates : incidence and risk factors. J Oral Maxillofac Surg 64:995-996

6. Pazianas M, Miller P, Blumental WA, Bernal M, Kothawala P (2007) A review of the literature on osteonecrosis of the jaw in patients with osteoporosis treated with oral bisphosphonates : prevalence, risk factors, and clinical characteristics. Clin Therapeut 29:1548-1558

7. Cartsos VM, Zhu S, Zavras AI (2008) Bisphosphonate use and the risk of adverse jaw outcomes. A medical claims study of 714, 217 people. J Am Dent Ass 139:23-30

8. Marx RE, Cillo JE Jr, Ulloa JJ (2007) Oral bisphosphonate-induced osteonecrosis: risk factors. Prediction of risk using serum CTX testing, prevention, and treatment. J Oral Maxillofac Surg 65:2377-410

9. Takaishi Y, Ikeo T, Miki T, Nishizawa Y, Morii H (2003) Suppression of alveolar bone resorption by periodontal disease : 4 to 5 year follow-up of 4 patients. J Int Med Res 31:575-584

10. Takaishi Y, Ikeo T, Miki T, Morii H (2005) Correlations between periodontitis and loss of mandibular bone in relation to systemic bone changes in postmenopausal Japanese women. Osteopor Int 16:1875-1882 